INPLASY

PROTOCOL

To cite: Xu et al. Effectiveness and safety of

Electroacupuncture in treating

Parkinson's disease: protocol

of a systematic review and

meta-analyses. Inplasy

protocol 202120031. doi:

10.37766/inplasy2021.2.0031

Received: 07 February 2021

Published: 08 February 2021

Corresponding author:

Wei Xu

xuwei20190502@163.com

Author Affiliation:

The Affiliated hospital-Jiangxi

University of TCM

Support: 81860877, 81660821, $20181 B B G 7004$.

Review Stage at time of this submission: The review has not yet started.

Conflicts of interest:

None declared.

\section{Effectiveness and safety of Electroacupuncture in treating Parkinson's disease: protocol of a systematic review and meta-analyses}

Xu, W1ㅍ Jiao, L2; Li, J33.

Review question / Objective: This study comprehensively searched the literature to further systematically evaluate The efficacy and safety of Electroacupuncture in treating Parkinson's disease, with a view to clinically treating Parkinson's disease, alleviating its related clinical symptoms and preventing its further development, and providing the latest evidence-based medical evidence.

Condition being studied: Parkinson's disease (PD) is an Extrapyramidal Disease mainly characterized by static tremor, myotonia, bradykinesia and postural gait disorder.As China's population ages, the number of people suffering from Parkinson's disease is increasing.Since there are many side effects of western medicine for Parkinson's patients, and the high price of the drugs make it difficult for many patients to adhere to treat.At present, many clinical studies have shown that electroacupuncture is effective in treating Parkinson's disease.Therefore, this systematic review aims to explore the effectiveness and safety of electroacupuncture in the treatment of Parkinson's disease.

INPLASY registration number: This protocol was registered with the International Platform of Registered Systematic Review and Meta-Analysis Protocols (INPLASY) on 08 February 2021 and was last updated on 08 February 2021 (registration number INPLASY202120031).

\section{INTRODUCTION}

Review question / Objective: This study comprehensively searched the literature to further systematically evaluate The efficacy and safety of Electroacupuncture in treating Parkinson's disease, with a view to clinically treating Parkinson's disease, alleviating its related clinical symptoms and preventing its further development, and providing the latest evidence-based medical evidence.

Condition being studied: Parkinson's disease (PD) is an Extrapyramidal Disease mainly characterized by static tremor, 
myotonia, bradykinesia and postural gait disorder. As China's population ages, the number of people suffering from Parkinson's disease is increasing. Since there are many side effects of western medicine for Parkinson's patients, and the high price of the drugs make it difficult for many patients to adhere to treat. At present, many clinical studies have shown that electroacupuncture is effective in treating Parkinson's disease. Therefore, this systematic review aims to explore the effectiveness and safety of electroacupuncture in the treatment of Parkinson's disease.

\section{METHODS}

Search strategy: The following databases will be searched: PubMed, Embase, Medline, Cochrane Database of Systematic Reviews, Chinese Biomedical Literatures Database(CBM), China National Knowledge Infrastructure(CNKI), Chinese Scientific Journal Database(VIP), Wang Fang Database(WF) from inception to January 2021. The main subject terms searched: "electroacupuncture","Parkinso n". Pubmed's search strategy is shown in Table 1; Other databases' search strategies will be adjusted according to each database.

Participant or population: Participants in this study were required to meet the international recognized diagnostic criteria for PD. There is no restriction on age, sex, nationality, but the PD participants will be excluded if one accompanied by serious cardiovascular and cerebrovascular diseases, and life-threatening complications.

Intervention: PD participants in the test group must be treated with electroacupuncture as the main regimen (either in combination with other treatments or alone) and the control group must be treated with nonelectroacupuncture therapy.

Comparator: 1. Electroacupuncture therapy vs no treatment; 2. Electroacupuncture therapy vs placebo; 3 . Electroacupuncture therapy vs sham acupuncture; 4 . Electroacupuncture therapy vs symptomatic or active treatment.

Study designs to be included: A randomized controlled trial (RCT) study on electroacupuncture in treating Parkinson's disease, published in any language.

Eligibility criteria: Types of study: All randomized controlled trials (RCT s) study on electroacupuncture in treating Parkinson's disease. Others such as case reports, animal experiments, non-RCTs, or RCT protocol will be excluded.

Information sources: PubMed, Web of Science, the Cochrane Database, EMBASE, China Knowledge Network (CNKI),Wanfang Data Knowledge Service Platform, VIP Chinese Science and Technology Periodical Database (VIP) and China Biomedical Literature (CBM) Database.

Main outcome(s): The total effective rate and the total symptom score were the main outcomes. The total symptom score will be based on the scale of UPDRS(including the score of spirit, behavior, emotion, daily activities, motor function, motor complications), the Webster scale.

Additional outcome(s): 1. Quality of life questionnaire (QLQ) ; 2. The score of HAMD; 3. Laboratory inspection indicators: the level of the content of lipid peroxidase, Superoxide dismutase activity in plasma and erythrocyte; 4. recurrence rate; 5. Adverse events.

Data management: The included literature will be conducted as follows: Firstly, imported the retrieved literature into NoteExpress 3.1software, and eliminate the duplicate literature in the document manager;Secondly, remove the literature not relevant to this study by reading the title and abstract of literature one by one; Thirdly, download the remaining articles in sequence and read the full text; Finally, according to the inclusion and exclusion 
criteria required in this article, then the final paper is determined.

Quality assessment / Risk of bias analysis: Two reviewers performed rigorous methodological quality evaluation of the included studies with reference to the Cochrane Collaboration Bias Risk Assessment Tool for the extracted methodological features.

Strategy of data synthesis: Meta analysis was performed using RevMan5.3 provided by the Cochrane collaboration network. Relative risk (RR) was used for the two categorical variables, and mean difference(MD) was used for the continuous variables. Both were expressed with $95 \%$ confidence intervals $(\mathrm{Cl})$. The heterogeneity test between the results of the included studies was performed using the $\mathrm{I}^{2}$ test.The $I^{2}$ value reflects the proportion of the total variation in the effect size due to the existence of heterogeneity. $\left(I^{2}>50 \%\right)$, indicating that heterogeneity is more obvious. If there is no obvious heterogeneity between the research results (I $50 \%$ ), the source of the heterogeneity is analyzed first, which may lead to heterogeneity Factors for subgroup analysis. If statistical heterogeneity exists in each subgroup without clinical heterogeneity, a random effects model is used for analysis. If the heterogeneity is too large and the results cannot be combined, a descriptive analysis is used and a sensitivity analysis is performed if necessary.

Subgroup analysis: Subgroup analysis will be implemented based on different control measures if there is a large heterogeneity.

Sensitivity analysis: STATA 14.0 software will be used for sensitivity analysis, and the sensitivity analysis is performed to assess the reliability of the meta-analysis.

Language: No limited.

Country(ies) involved: China.

Other relevant information: None.
Keywords: Electroacupuncture; Parkinson's disease; Randomized controlled trial; Systematic review

Contributions of each author:

Author 1 - Wei Xu.

Email: xuwei20190502@163.com

Author 2 - Lin Jiao.

Email: j10809@126.com

Author 3 - Li, J. 